\title{
THE MORSE INDEX THEOREM WHERE THE ENDS ARE SUBMANIFOLDS
}

\author{
DIANE KALISH
}

\begin{abstract}
In this paper the Morse Index Theorem is proven in the case where submanifolds $P$ and $Q$ are at the endpoints of a geodesic, $\gamma$. At $\gamma$, the index of the Hessian of the energy function defined on paths joining $P$ and $Q$ is computed using $P$-focal points, and a calculation at the endpoint of $\gamma$, involving the second fundamental form of $Q$.
\end{abstract}

1. Introduction. Let $M$ be a complete Riemannian manifold with submanifolds $P$ and $Q$. The energy function $E$ is defined on the space $\Omega(M ; P, Q)$ of piecewise $C^{\infty}$ paths joining $P$ and $Q$. A path $\gamma \in \Omega(M ; P, Q)$ is a critical point for $E$ when $\gamma$ is a geodesic intersecting $P$ and $Q$ orthogonally. The tangent space, $T \Omega_{\gamma}$, consists of piecewise $C^{\infty}$ vector fields along $\gamma$ with initial and final vectors tangential to $P$ and $Q$, respectively. A symmetric bilinear map, $I$, is defined on $T \Omega_{\gamma} \times T \Omega_{\gamma}$ to $R$ and is called the Morse index form.

When $Q$ is a point, the Morse Index Theorem yields the index of $I$ as the sum of the $P$-focal points along $\gamma$ counted with multiplicities. Both Ambrose [1] and Bolton [2] have proven index theorems in the general case, where $Q$ is a submanifold. Ambrose defines a " $(P, Q)$ conjugate point," while Bolton uses the notion of a signed $(P, Q)$ focal point which is employed in the calculations of the index of $I$.

In this paper the index of a critical point $\gamma$ is found using $P$-focal points and a computation at the endpoint of $\gamma$ contained in $Q$, involving the second fundamental form of $Q$ with respect to $\dot{\gamma}$. This method allows for a simpler proof of the theorem as well as an easy computation of the index of geodesics in many spaces. In a paper to follow this one, the homotopy type of some path spaces joining submanifolds on a Riemannian manifold have been computed. This result is obtained, with some minor modifications, by following the proof in Milnor [4, pp. 88-95].

I would like to thank my advisor, Professor Edgar Feldman, for his invaluable advice, guidance, and encouragement. I also wish to thank the referee for some excellent suggestions which made my paper more concise.

2. Definitions. $M$ is a complete Riemannian manifold of dimension $d$ with the Levi-Civita connection.

$\gamma(t), t \in[0, T]$, is a geodesic in $M$.

$P$ and $Q$ are submanifolds of $M$ with $\gamma(0) \in P ; \gamma^{\prime}(0) \perp P_{\gamma(0)} ; \gamma(T) \in Q ; \gamma^{\prime}(T)$ $\perp Q_{\gamma(T)}$.

$r$ is the dimension of $Q_{\gamma(T)}, 0 \leq r<d$.

Received by the editors November 4, 1986 and, in revised form, June 2, 1987.

1980 Mathematics Subject Classification (1985 Revision). Primary 58E05.

This paper is part of the author's doctoral dissertation at the Graduate Center of the City University of the City of New York. 
$H$ is the linear space of continuous piecewise $C^{\infty}$ vector fields along $\gamma$ which are orthogonal to $\gamma$ and whose initial and final vectors are in $P_{\gamma(0)}$ and $Q_{\gamma(T)}$, respectively. Then,

$$
H=\left\{V(t)=\sum_{i=1}^{d-1} h_{i}(t) E_{i}(t) \text { with } V_{(0)} \in P_{\gamma(0)} ; V(T) \in Q_{\gamma(T)}\right\}
$$

where $E_{1}, \ldots, E_{d-1}$ are orthonormal parallel vector fields along $\gamma$ and orthogonal to $\gamma$, and $h_{1}, \ldots, h_{d-1}$ are real valued continuous piecewise $C^{\infty}$ functions defined on $[0, T]$.

A Jacobi field $X$ is a vector field along $\gamma$ which satisfies the differential equation $X^{\prime \prime}-R X=0$, where $R X=R\left(\gamma^{\prime}, X\right) \gamma^{\prime}$ is the curvature tensor of the Levi-Civita connection.

A $P$-Jacobi field is a Jacobi field which is orthogonal to $\gamma$ with $J(0) \in P_{\gamma(0)}$ and $J^{\prime}(0)-S_{0} J(0) \perp P_{\gamma(0)}$, where $S_{0}$ is the second fundamental form of $P$ at $\gamma(0)$ with respect to $\gamma^{\prime}(0)$.

$J_{1}, \ldots, J_{d-1}$ are $d-1$ linearly independent $P$-Jacobi fields which span the space of $P$-Jacobi fields.

$B$ is the set of all $X \in H$ such that

$$
X(t)=\sum_{i=1}^{d-1} f_{i}(t) J_{i}(t) \quad \text { with } X(T)=0,
$$

and $f_{1}, \ldots, f_{d-1}$ are real valued continuous piecewise $C^{\infty}$ functions defined on $[0, T]$.

$I$ is a symmetric bilinear map from $H \times H$ to $R$ defined as follows.

$$
\begin{aligned}
I(X, Y)= & \int_{0}^{T}\left\langle R X(t)-X^{\prime \prime}(t), Y(t)\right\rangle d t \\
& +\sum_{i}\left\langle X^{\prime}\left(p_{i}^{-}\right)-X^{\prime}\left(p_{i}^{+}\right), Y\left(p_{i}\right)\right\rangle+\left.\left\langle X^{\prime}(t)-S_{t} X(t), Y(t)\right\rangle\right|_{0} ^{T}
\end{aligned}
$$

where $p_{i}$ is a point of discontinuity of $X^{\prime}$ in $(0, T) ; S_{0}$ is the second fundamental form of $P$ at $\gamma(0)$ with respect to $\gamma^{\prime}(0)$; and $S_{T}$ is the second fundamental form of $Q$ at $\gamma(T)$ with respect to $\gamma^{\prime}(T)$.

A $P$-focal point is a point $\gamma(t), t \in(0, T]$, for which there exists a nonzero $P$-Jacobi field which vanishes at $t$.

The multiplicity, $m$, of the $P$-focal point $\gamma(t)$ is the dimension of the space of $P$-Jacobi fields which vanish at $t$.

$A$ is a symmetric bilinear map defined on the space spanned by $J_{1}, \ldots, J_{d-1}$ whose value at $T$ is contained in $Q_{\gamma(T)}$ and is defined as follows:

$$
A(V, W)=\left\langle V^{\prime}(T)-S_{T} V(T), W(T)\right\rangle .
$$

\section{The Index Theorem.}

THEOREM (MORSE INDEX THEOREM WITH VARIABLE ENDPOINTS). The index of $I$ is equal to the number of points $\gamma(t)$, with $0<t<T$, such that $\gamma(t)$ is a $P$-focal point; each such $P$-focal point counted with its multiplicity, plus the index of $A$. (Assume $\gamma(T)$ is not a P-focal point.) 
The above theorem states

$$
i(I)=\sum_{i=1}^{k} m_{i}+i(A),
$$

when $\gamma(T)$ is not a $P$-focal point, where $i(I)=\operatorname{index}$ of $I ; i(A)=\operatorname{index}$ of $A ; m_{i}$ is the multiplicity of $\gamma\left(t_{i}\right)$; and $\gamma\left(t_{1}\right), \ldots, \gamma\left(t_{k}\right)$ are the set of $P$-focal points along $\gamma,\left(0<t_{1}<\cdots<t_{k}<T\right)$.

Our aim will be to write $H=B \oplus B^{C}$, where $I$ is positive on $B$. (For definition of $B$ see $\S 2$.) We will show that $B^{C}$ is a finite dimensional space and construct a subspace of $B^{C}$ on which $I$ is negative definite and whose dimension is equal to or greater than any other subspace of $H$ on which $I$ is negative definite. This will yield $i(I)$.

REMARK. The subspace $B$ can be characterized as the set of all vector fields in $H$ whose values at the $P$-focal points are in the span of $J_{1}, \ldots, J_{d-1}$ and whose value at $T$ is zero.

This follows from the fact that any broken $C^{\infty}$ vector field which can be expressed as $\sum_{i=1}^{d-1} f_{i}(t) E_{i}(t)$ and is in the span of $J_{1}\left(t_{i}\right), \ldots, J_{d-1}\left(t_{i}\right)$ at all $P$-focal points $t_{i}$ can also be written as $\sum_{i=1}^{d-1} g_{i}(t) J_{i}(t)$ for broken $C^{\infty}$ functions $g_{i}$ [3, p. 231].

The next two definitions will yield $\left(\sum_{i=1}^{k} m_{i}+r\right)$ linearly independent elements of $H$ whose span will be denoted by $B^{C}$.

DEFinition OF $K_{2}$. Since $T$ is not a $P$-focal point, we can choose $r$ linearly independent $P$-Jacobi fields $K_{1}, \ldots, K_{r}$ with the following properties: (1) $K_{1}(T), \ldots, K_{r}(T) \operatorname{span} Q_{\gamma(T)} ;(2) A$ is negative definite on the span of $K_{1}, \ldots, K_{N}$ where $N=\operatorname{index} A$ and $N \leq r$; and (3) $A$ is positive $(\geq 0)$ on the span of $K_{n+1}, \ldots, K_{r}$. (Recall that $A$ was defined as a symmetric bilinear map on those $P$-Jacobi fields which, when evaluated at $T$, lie in $Q_{\gamma(T)}$. The dimension of this space is $r$.)

DEFINITION OF $V_{i}^{j_{i}}$. Consider the $P$-focal point $\gamma\left(t_{i}\right)$ with multiplicity $m_{i}$. Let $Y_{i}^{1}, \ldots, Y_{i}^{m_{i}}$ be $m_{i}$ linearly independent $P$-Jacobi fields such that

(1) $Y_{i}^{j_{i}}\left(t_{i}\right)=0$ for $j_{i}=1, \ldots, m_{i}$,

(2) $\left\{Y_{i}^{\prime j_{i}}\left(t_{i}\right)\right\}$ for $j_{i}=1, \ldots, m_{i}$, form an orthonormal set.

Let $\tilde{Z}_{i}^{j_{i}}$ be parallel vector fields along $\gamma$ such that

$$
\tilde{Z}_{i}^{j_{i}}\left(t_{i}\right)=-Y_{i}^{\prime j_{i}}\left(t_{i}\right) \text { for } j_{i}=1, \ldots, m_{i} .
$$

Let $\phi_{i}:[0, T] \rightarrow R$ be a $C^{\infty}$ function such that $(1) \phi_{i}\left(t_{i}\right)=1 ;(2) \phi_{i}\left(t_{i}\right)$ has small support about $t_{i}$; and (3) $0 \leq \phi_{i}(t) \leq 1$. Let

$$
Z_{i}^{j_{i}}(t)=\phi_{i}(t) \tilde{Z}_{i}^{j_{i}}(t) ; \quad i=1, \ldots, m_{i}
$$

Let

$$
V_{i}^{j_{i}}(t)= \begin{cases}Y_{i}^{j_{i}}(t)+\lambda Z_{i}^{j_{i}}(t) & \text { for } 0 \leq t \leq t_{i} \\ \lambda Z_{i}^{j_{i}}(t) & \text { for } t_{i} \leq t \leq T\end{cases}
$$

where $\lambda>0$.

(Note: It follows from (1) and (2) in the definition of $V_{i}^{j_{i}}$ that

$$
\left.\left(\operatorname{span}\left[Y_{i}^{\prime 1}\left(t_{i}\right), \ldots, Y_{i}^{\prime m_{i}}\left(t_{i}\right)\right]\right)^{\perp}=\operatorname{span}\left(J_{1}\left(t_{i}\right), J_{2}\left(t_{i}\right), \ldots, J_{d-1}\left(t_{i}\right), \gamma^{\prime}\left(t_{i}\right)\right) .\right)
$$



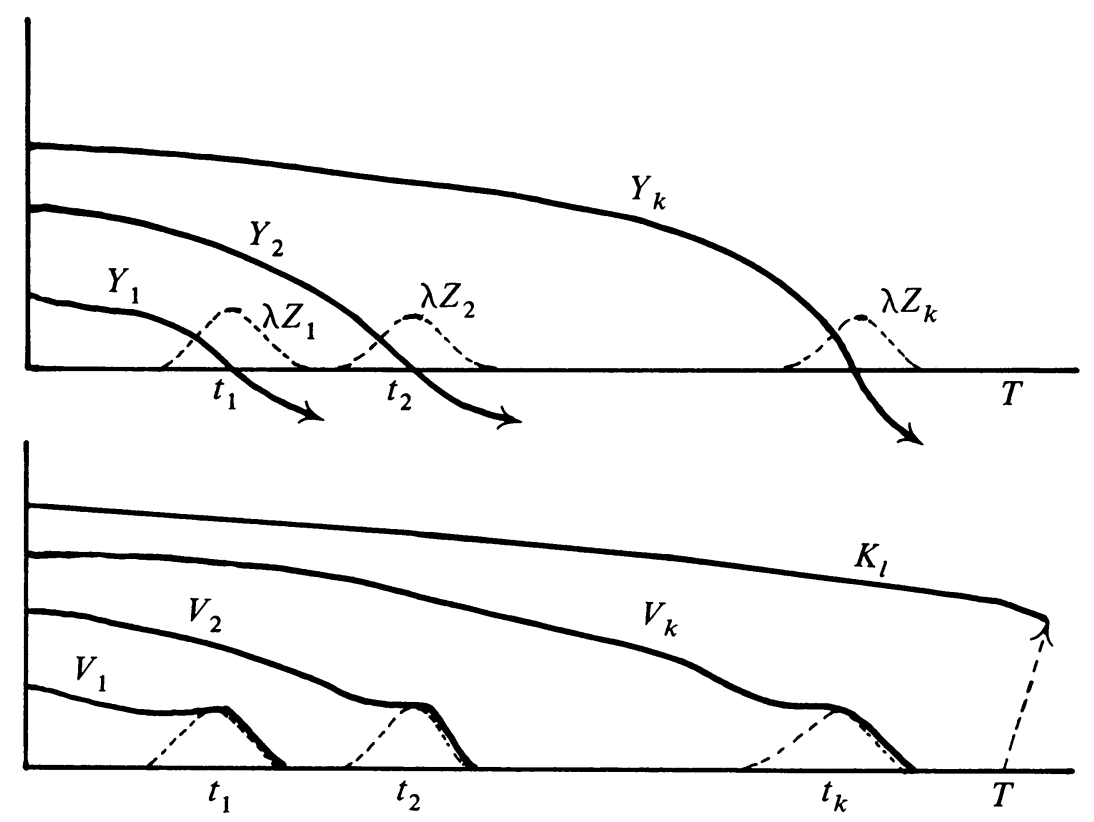

FIGURE 1

DEFinition of $B^{C}$. Let $B^{C}$ denote the span of the vectors $V_{i}^{j_{i}}(i=1, \ldots, k$; $\left.j_{i}=1, \ldots, m_{i}\right)$ and $K_{l}(l=1, \ldots, r)$.

Claim. (1) The dimension of $B^{C}$ is $\sum_{i=1}^{k} m_{i}+r$,

(2) $B^{C} \cap B=0$.

The claim follows from the definitions of $B$ and $B^{c}$, by making the support of each $\phi_{i}$ small enough, and by looking at Figure 1.

LEMMA 1. $H=B \oplus B^{c}$.

Proof. Let $x \in H$. There is a $c \in B^{c}$ such that $x-c \in B$. This can be accomplished by choosing $c$ equal to a linear combination of elements in $B^{c}$, so that at $P$-focal point the value of $x-c$ lies in the span of the $P$-Jacobi fields and at $T$ is equal to zero. Thus $x-c \in B$. Since $B \cap B^{c}=0$, we have Lemma 1 .

Lemma 2 will show $I$ is positive on $B$ and Lemma 3 will exhibit a subspace of $B^{C}$ on which $I$ is negative definite.

LEMMA 2. $I(V, V) \geq 0$ for $V \in B$.

Proof. Let $V \in B$. Then

$$
V=\sum_{i=1}^{d-1} f_{i} J_{i}
$$


and

$$
\begin{aligned}
I(V, V)= & \left\langle\sum_{i=1}^{d-1} f_{i}(T) J_{i}^{\prime}(T)-S_{T}[V(T)], V(T)\right\rangle \\
& \left.\left.+\int_{0}^{T}\left\langle\sum f_{i}^{\prime}(t) J_{i}(t), \sum f_{i}^{\prime}(t) J_{i}\right) t\right)\right\rangle d t \\
= & \int_{0}^{T}\left\langle\sum f_{i}^{\prime}(t) J_{i}(t), \sum f_{i}^{\prime}(t) J_{i}(t)\right\rangle d t \quad(\text { since } V(T)=0) \\
\geq & 0 .
\end{aligned}
$$

For the first equality, see [3, p. 229]. This proves Lemma 2.

LEMMA 3.

$$
\operatorname{index}\left(I_{\mid B^{C}}\right)=\sum_{i=1}^{k} m_{i}+\operatorname{index}(A) .
$$

ProOF. $K_{1}, \ldots, K_{r}$ were chosen to be $r$ linearly independent $P$-Jacobi fields such that $A$ is negative definite $(<0)$ on the span of $K_{1}, \ldots, K_{N}$ and positive $(\geq 0)$ on $K_{N+1}, \ldots, K_{r}, N=\operatorname{index} A$.

We wish to show that $I$ is negative definite on the span of $\left\{V_{i}^{j_{i}}\right\}_{i=1, \ldots, k} ; j_{i}=1, \ldots, m_{k}$ and $K_{1}, \ldots, K_{N}$, and that $I$ is positive on the span of $K_{N+1}, \ldots, K_{r}$.

$$
\begin{aligned}
I\left(\sum_{i, j_{i}} \alpha_{i}^{j_{i}} V_{i}^{j_{i}}+\sum_{l=1}^{N} \beta_{l} K_{l}\right) \\
=I\left(\sum \alpha_{i}^{j_{i}} V_{i}^{j_{i}}\right) \\
+2 I\left(\sum_{i, j_{i}} \alpha_{i}^{j_{i}} V_{i}^{j_{i}}, \sum_{l=1}^{N} \beta_{l} K_{l}\right) \\
+I\left(\sum_{l=1}^{N} \beta_{l} K_{l}\right) .
\end{aligned}
$$

The computation of $(3)$ yields $I\left(\sum \beta_{l} K_{l}\right)<0$.

PROOF. Let

$$
K=\sum_{l=1}^{N} \beta_{l} K_{l}
$$

Then

$$
\begin{aligned}
I(K)= & \int_{0}^{T}\left\langle R K-K^{\prime \prime}, K\right\rangle d t+\sum_{\substack{\text { jumps } \\
\text { of } K^{\prime}}}\left\langle K^{\prime}\left(p_{i}^{-}\right)-K^{\prime}\left(p_{i}^{+}\right), V_{i}^{j_{i}}\left(p_{i}\right)\right\rangle \\
& +\left.\left\langle K^{\prime}(t)-S_{t} K(t), K(t)\right\rangle\right|_{0} ^{T}=\left\langle K^{\prime}(T)-S_{T} K(T), K(T)\right\rangle .
\end{aligned}
$$

This follows from the fact that $K$ is a $P$-Jacobi field which is smooth and satisfies $R K-K^{\prime \prime}=0$ and $K^{\prime}(0)-S_{0} K(0) \perp P_{\gamma(0)}$.

So $I(K)=\left\langle K^{\prime}(T)-S_{T} K(T), K(T)\right\rangle=A(K)<0$, since $A$ is negative definite on the span of $K_{1}, \ldots, K_{N}$. 
The computation of (2) yields

$$
I\left(\sum \alpha_{i}^{j_{i}} V_{i}^{j_{i}}, \sum \beta_{l} K_{l}\right)=0
$$

Proof. When (2) is expanded we get linear combinations of terms of the form $I\left(V_{i}^{j_{i}}, K_{l}\right)$.

$$
\begin{aligned}
I\left(V_{i}^{j_{i}}, K_{l}\right)= & \int_{0}^{T}\left\langle R K_{l}-K_{l}^{\prime \prime}, V_{i}^{j_{i}}\right\rangle d t+\sum_{\substack{\text { jumps } \\
\text { of } K^{\prime}}}\left\langle K^{\prime}\left(p_{i}^{-}\right)-K^{\prime}\left(p_{i}^{+}\right), K\left(p_{i}\right)\right\rangle \\
& +\left.\left\langle K_{l}^{\prime}(t)-S_{t} K_{l}(t), V_{i}^{j_{i}}(t)\right\rangle\right|_{0} ^{T}=0,
\end{aligned}
$$

since $K_{l}$ is a $P$-Jacobi field, $K_{l}^{\prime}(0)-S_{0} K(0) \perp P_{\gamma(0)}\left(V_{i}^{j_{i}}(0) \in P_{\gamma(0)}\right)$, and $V_{i}^{j_{i}}(T)=$ 0 .

The computation of (1) yields $I\left(\sum \alpha_{i}^{j_{i}} V_{i}^{j_{i}}\right)<0$.

PROOF. Let

$$
\hat{Y}_{i}^{j}= \begin{cases}Y_{i}^{j}(t), & 0 \leq t \leq t_{i}, \\ 0, & t_{i} \leq t \leq T .\end{cases}
$$

Then $V_{i}^{j}(t)=\hat{Y}_{i}^{j}(t)+\lambda Z_{i}^{j}(t) ; t \in[0, T]$.

$$
\begin{gathered}
I\left(V_{i}^{j}, V_{h}^{l}\right)=I\left(\hat{Y}_{i}^{j}+\lambda Z_{i}^{j}, \hat{Y}_{h}^{l}+\lambda Z_{h}^{l}\right) \\
= \begin{cases}I\left(\hat{Y}_{i}^{j}, \hat{Y}_{h}^{l}\right) & \text { (a) } \\
+\lambda^{2} I\left(Z_{i}^{j}, Z_{h}^{l}\right) & \text { (b) } \\
+\lambda I\left(Z_{i}^{j}, \hat{Y}_{h}^{l}\right)+\lambda I\left(\hat{Y}_{i}^{j}, Z_{h}^{l}\right) & \text { (c). }\end{cases} \\
(\mathrm{a})=I\left(\hat{Y}_{i}^{j}, \hat{Y}_{h}^{l}\right)=\int_{0}^{T}\left\langle R \hat{Y}_{i}^{j}-\hat{Y}_{i}^{\prime \prime j}, \hat{Y}_{h}^{l}\right\rangle d t+\left\langle\hat{Y}_{i}^{\prime j}\left(t_{i}^{-}\right)-\hat{Y}_{i}^{\prime j}\left(t_{i}^{+}\right), \hat{Y}_{h}^{l}\left(t_{i}\right)\right\rangle \\
+\left.\left\langle\hat{Y}_{i}^{\prime j}-S_{t} \hat{Y}_{i}^{j}, \hat{Y}_{h}^{l}\right\rangle\right|_{0} ^{T}=0, \quad \text { when } h \leq i .
\end{gathered}
$$

This follows from $\hat{Y}_{i}^{\prime \prime j}-R \hat{Y}_{i}^{j}=0, \hat{Y}_{h}^{l}\left(t_{i}\right)=0, \hat{Y}_{h}^{l}(T)=0$, and $\hat{Y}_{i}^{\prime j}(0)-S_{0} \hat{Y}_{i}^{j}(0) \perp$ $P_{\gamma(0)}$. Since $I\left(\hat{Y}_{i}^{j}, \hat{Y}_{h}^{l}\right)=I\left(\hat{Y}_{h}^{l}, \hat{Y}_{i}^{j}\right)$, the same argument works for $i \leq h$.

(c) $=-2 \lambda \delta_{i h} \delta_{j l}$, which is shown to be true as follows. For $i \neq h$, we get

$$
\begin{aligned}
I\left(Z_{i}^{j}, \hat{Y}_{h}^{l}\right)= & \int_{0}^{T}\left\langle R \hat{Y}_{h}^{l}-\hat{Y}_{h}^{\prime \prime l}, Z_{i}^{j}\right\rangle d t+\left\langle\hat{Y}_{h}^{\prime l}\left(t_{h}^{-}\right)-\hat{Y}_{h}^{\prime l}\left(t_{h}^{+}\right), Z_{i}^{j}\left(t_{h}\right)\right\rangle \\
& +\left.\left\langle\hat{Y}_{h}^{\prime l}(t)-S_{t} \hat{Y}_{h}^{l}(t), Z_{i}^{j}(t)\right\rangle\right|_{0} ^{T}=0,
\end{aligned}
$$

since $\hat{Y}_{h}^{l}$ is a $P$-Jacobi field on $\left[0, t_{h}\right]$,

$$
Z_{i}^{j}(0)=Z_{i}^{j}(T)=0 \text { and } Z_{i}^{j}\left(t_{h}\right)=0
$$

when the support of $\phi_{i}$ is small enough.

For $i=h$, we get the same as above except at $t_{i}$,

$$
\begin{aligned}
I\left(Z_{i}^{j}, \hat{Y}_{i}^{l}\right)= & \left\langle\hat{Y}_{i}^{\prime l}\left(t_{i}^{-}\right)-\hat{Y}_{i}^{\prime l}\left(t_{i}^{+}\right), Z_{i}^{j}\left(t_{i}\right)\right\rangle \\
= & \left\langle\hat{Y}_{i}^{\prime l}\left(t_{i}^{-}\right), Z_{i}^{j}\left(t_{i}\right)\right\rangle \quad\left(\text { since } \hat{Y}_{i}^{l}(t)=0 \text { for } t \in\left[t_{i}, T\right]\right) \\
= & \left\langle Y_{i}^{\prime l}\left(t_{i}\right),-Y_{i}^{\prime j}\left(t_{i}\right)\right\rangle \\
= & -\delta_{j l}\left\|Y_{i}^{\prime j}\left(t_{i}\right)\right\|^{2} \\
& \quad\left(\text { since }\left\{Y_{i}^{\prime 1}, Y_{i}^{\prime 2}, \ldots, Y_{i}^{\prime m_{i}}\right\} \text { is an orthonormal set evaluated at } t_{i}\right) \\
= & -\delta_{j l} .
\end{aligned}
$$


Therefore, $\lambda I\left(Z_{i}^{j}, \hat{Y}_{h}^{l}\right)+\lambda I\left(\hat{Y}_{i}^{j}, Z_{h}^{l}\right)=-2 \lambda \delta_{i h} \delta_{j l}$.

Putting together the results from (a), (b), and (c), we have

$$
I\left(V_{i}^{j}, V_{h}^{l}\right)=\lambda^{2} I\left(Z_{i}^{j}, Z_{h}^{l}\right)-2 \lambda \delta_{i h} \delta_{j l} .
$$

Notation. Let $A$ be the $\left(\sum m_{i}\right) \times\left(\sum m_{i}\right)$ symmetric matrix $\left(I\left(Z_{i}^{j_{i}}, Z_{k}^{j_{k}}\right)\right)$. If $X=\sum a_{i}^{j_{i}} V_{i}^{j_{i}}, Y=\sum b_{i}^{j_{i}} V_{i}^{j_{i}}$ let

$$
\langle X, Y\rangle=\sum a_{i}^{j_{i}} b_{i}^{j_{i}} \text { and }\|X\|^{2}=\langle X, X\rangle .
$$

Then for $X=\sum_{i, j_{i}} a_{i}^{j_{i}} V_{i}^{j_{i}}$ we have

$$
I(X, X)=\lambda^{2}\langle A X, X\rangle-2 \lambda\langle X, X\rangle .
$$

If $A=0, I(X, X)<0$ for $\lambda>0, X \neq 0$. If $A \neq 0$, $\|A\| \neq 0$, let $0<\lambda<2 /\|A\|$. Then

$$
\begin{aligned}
I(X, X) & =\lambda^{2}\langle A X, X\rangle-2 \lambda\langle X, X\rangle \leq \lambda^{2}\|A X\|\|X\|-2 \lambda\|X\|^{2} \\
& \leq \lambda^{2}\|A\|\|X\|^{2}-2 \lambda\|X\|^{2} \\
& <\lambda \frac{2}{\|A\|}\|A\|\|X\|^{2}-2 \lambda\|X\|^{2}=0 .
\end{aligned}
$$

This gives

$$
I\left(\sum \alpha_{i}^{j_{i}} V_{i}^{j_{i}}, \sum \alpha_{i}^{j_{i}} V_{i}^{j_{i}}\right)<0 \text { for } \sum \alpha_{i}^{j_{i}} V_{i}^{j_{i}} \neq 0 .
$$

Thus we have the computation of (1).

The results from (1), (2), and (3) show that $I$ is negative definite on the span of $\left\{V_{i}^{j_{i}}, K_{1}, \ldots, K_{N}\right\}_{i=1, \ldots, k} ; j_{i}=1, \ldots, m_{i}$.

In order to finish proving Lemma 3 we need to show $I$ is positive $(\geq 0)$ on the span of $K_{N+1}, \ldots, K_{r}$.

$$
I\left(\sum_{l=N+1}^{r} b_{l} K_{l}\right)=A\left(\sum_{l=N+1}^{r} b_{l} K_{l}\right) \geq 0,
$$

since $A$ is positive on the span of $K_{N+1}, \ldots, K_{r}$ and $\sum b_{l} K_{l}$ is a $P$-Jacobi field. This proves Lemma 3.

DEFINITION OF $B_{-}^{c}, B_{+}^{c}$. Let

$$
B_{-}^{c}=\operatorname{Span}\left(K_{1}, \ldots, K_{N}\right) \quad \text { and } \quad B_{+}^{c}=\operatorname{Span}\left(K_{N+1}, \ldots, K_{r}\right) .
$$

From Lemma 3 we have that $\left.I\right|_{B_{-}^{c}}<0$ and $\left.I\right|_{B_{+}^{c}} \geq 0$, while from Lemma 2 we have that $\left.I\right|_{B} \geq 0$.

Write $H=B \oplus B_{+}^{c} \oplus B_{-}^{c}$. In order to complete the Index Theorem we need to show that $I\left(B, B_{+}^{c}\right)=0$, which will follow if

$$
I\left(K_{j}, \sum f_{i} J_{i}\right)=0 \text { for } j=N+1, \ldots, r, i=1, \ldots, d-1 .
$$

This is true since $K_{j}$ is a $P$-Jacobi field, $S_{0} K_{j}(0)-K_{j}^{\prime}(0) \perp\left(\sum f_{i} J_{i}\right)(0)$ and $\left(\sum f_{i} J_{i}\right)(T)=0$.

We therefore have a subspace $B_{-}^{c}$ of $H$ on which $I$ is negative definite and whose dimension $\sum_{i=1}^{k} m_{i}+i(A)$ is the maximum value $I$ can attain on any subspace of $H$. Thus the Index Theorem is proven. 
4. Remarks. 1. The proof of the Index Theorem is symmetric with respect to the $P$ and $Q$ submanifolds at the ends of the geodesic. That is, we can use $Q$-Jacobi fields and $Q$-focal points to prove the theorem.

2. If $\gamma(T)$ is a $P$-focal point, then the proof of the Index Theorem is still valid when

(a) $Q_{\gamma(T)}$ is contained in the span of the $P$-Jacobi fields or when

(b) $\gamma(0)$ is not a $Q$-focal point, or if $P_{\gamma(0)}$ is contained in the span of the $Q$-Jacobi fields.

\section{REFERENCES}

1. W. Ambrose, The Index Theorem in Riemannian geometry, Ann. of Math. (2) 73 (1961), 49-86.

2. John Bolton, The Morse Index Theorem in the case of two variable end-points, J. Differential Geom. 12 (1977), 567-581.

3. Richard L. Bishop and Richard L. Crittenden, Geometry of manifolds, Academic Press, New York, 1964.

4. J. Milnor, Morse theory, Ann. of Math. Studies, no. 51, Princeton Univ. Press, Princeton, N.J., 1973.

Department of Mathematics, William Paterson College, Wayne, New JerSEY 07470 